\title{
Evidence of interstitial Cajal-like cells in the human gallbladder
}

\author{
Artur Pasternak ${ }^{1,2}$, Mariusz Gajda ${ }^{3}$, Krzysztof Gil ${ }^{4}$, Andrzej Matyja ${ }^{1}$, \\ Krzysztof A. Tomaszewski², Jerzy A. Walocha ${ }^{2}$, Jan Kulig ${ }^{1}$, Piotr Thor ${ }^{4}$ \\ ${ }^{1}$ First Department of General, Oncological and Gastrointestinal Surgery, Jagiellonian University \\ Medical College, Krakow, Poland \\ ${ }^{2}$ Department of Anatomy, Jagiellonian University Medical College, Krakow, Poland \\ ${ }^{3}$ Department of Histology, Jagiellonian University Medical College, Krakow, Poland \\ ${ }^{4}$ Department of Pathophysiology, Jagiellonian University Medical College, Krakow, Poland
}

\begin{abstract}
The aim of this study was to assess the presence of interstitial Cajal-like cells (ICLCs) in the human gallbladder and to determine their distinctive characteristics on the basis of double immunohistochemical staining. Gallbladder specimens were obtained from 30 patients subjected to elective laparoscopic cholecystectomy for symptomatic gallstone disease. Tissue samples were fixed in $4 \%$ phosphate-buffered paraformaldehyde, processed, embedded in paraffin, and, after sectioning, routinely stained with HE. Tissue antigens were retrieved using the heat-induced epitope retrieval (HIER) method. For simultaneous visualisation of two antigens, an indirect double immunofluorescence procedure was applied. ICLCs were defined as CD117-immunopositive and tryptase-immunonegative objects. They were predominantly fusiform in shape with sparse branches that were visible in some sections. ICLCs were observed throughout the organ including the gallbladder's fundus, body (corpus) and neck, being most numerous in the corpus. The ICLCs were detected almost exclusively within the muscularis propria and they were arranged parallel to smooth muscle cells. The following subpopulations of ICLCs were observed: ICLC-IM (intramuscular ICLCs) localised between smooth muscle fibres forming one muscle bundle; and ICLC-IB (interbundle ICLCs) localised within the connective tissue separating smooth muscle bundles. Thus, the presence of ICLCs in the human gallbladder was clearly identified, demonstrated by double immunohistochemistry which was found to be a reliable method for differentiating ICLCs from mast cells. (Folia Histochemica et Cytobiologica 2012, Vol. 50, No. 4, 581-585)
\end{abstract}

Key words: human gallbladder, interstitial Cajal-like cells, telocytes, immunofluorescence, c-Kit, tryptase, mast cells

\section{Introduction}

The term "interstitial cells of Cajal" (ICCs) refers to several types of cells located in the musculature of the gastrointestinal tract. In 1889, Santiago Ramon y Cajal, a Spanish neuropathologist, used the Golgi

Correspondence address: K. Tomaszewski, Department of Anatomy, Jagiellonian University Medical College,

Kopernika St. 12; 31-034 Krakow, Poland;

tel./fax: +48 1242295 11; e-mail: krtomaszewski@gmail.com technique and methylene blue staining to describe spindle-shaped or stellate cells associated with autonomic nerve endings of the intestine, and he proposed the term "cellules interstitielles" [1,2]. These cells were subsequently re-discovered approximately 40 years ago and they were successfully identified by electron microscopy and immunohistochemistry [3-5]. A characteristic feature of ICCs is the expression of transmembrane tyrosine kinase receptor proteins, including the c-Kit receptor (CD117), which enables their identification by immunohistochemical and molecular methods [6]. Signalling via c-Kit receptor is nec- 
essary for ICC development and phenotypic maintenance. ICCs are known to act as sources of spontaneous, electric slow waves responsible for paced contractions of the gastrointestinal tract ("pacemaker cells'). ICCs are also involved in the modulation of enteric neurotransmission [7]. Disturbances in gastrointestinal motility after the loss of, or damage to, ICCs have been widely reported in several clinical and pathological conditions, including gastroparesis, constipation, achalasia, Chagas disease, Hirschprung's disease, congenital hypertrophic pyloric stenosis, intestinal pseudo-obstructions, and diverticular disease of the colon [6, 8-10]. Many authors have investigated cells similar to ICCs located in other organs, including the pancreas, urether, urethra, bladder, blood vessels, male and female reproductive organs, mammary glands, placenta, heart, and lungs [8,11-17]. Cells that resemble archetypal enteric ICCs are referred to as interstitial Cajal-like cells (ICLCs). This term was proposed by Popescu and Faussone-Pellegrini in 2010 to be replaced by the entity — "telocytes".

The purpose of this study was to assess whether ICLCs occur in the human adult gallbladder and to determine their distinctive immunophenotypic characteristics on the basis of the double immunohistochemical staining protocol in gallbladders from patients operated for gallstone disease.

\section{Material and methods}

Gallbladders investigated in this study were obtained from 30 patients (eight males, mean age $51.9 \pm 10.7$ years and 22 females, mean age $52.9 \pm 15.1$ years) undergoing elective laparoscopic cholecystectomy for symptomatic gallstone disease. Cases of acute cholecystitis were excluded from the study. All patients were surgically treated in the First Department of General, Oncological and Gastrointestinal Surgery at the Jagiellonian University Medical College in 2010. The study was conducted in conformance with the moral, ethical, regulatory and scientific principles governing clinical research. All surgical samples were retrieved with the ethical approval of the Jagiellonian University Bioethical Committee (protocol number - KBET/30/B/2010) conforming to the Declaration of Helsinki guidelines.

Fresh gallbladder specimens were cut longitudinally, opened from the fundus to the neck and rinsed thoroughly with PBS (phosphate-buffered saline, 0.01 M, pH 7.4). Tissue samples were fixed in $4 \%$ phosphate-buffered paraformaldehyde, processed and embedded in paraffin. Serial sections were cut and mounted on poly-L-lysine-coated glass slides. The sections were deparaffinised in xylene, rehydrated through a graded series of alcohol, and transferred to PBS prior to staining. One part of the gallbladder sections was subjected to routine staining with haematoxylin and eosin (H\&E) and the other was used for immunohistochemical evaluation.

Immunoperoxidase staining of ICLC cells and mast cells. Tissue antigens were retrieved using the heat-induced epitope retrieval (HIER) method. The sections were incubated in a citrate buffer ( $\mathrm{pH}$ 6.0) for $15 \mathrm{~min}$ in a microwave oven $\left(250 \mathrm{~W}, 96^{\circ} \mathrm{C}\right)$ followed by cooling at room temperature. Endogenous peroxidase activity was blocked with $3 \%$ hydrogen peroxide in methanol for $20 \mathrm{~min}$. A pre-incubation step was performed with $5 \%$ normal goat serum and $0.5 \%$ Triton X-100 for $20 \mathrm{~min}$ to reduce non-specific binding and to increase penetration of the antibodies. The sections were incubated overnight at room temperature in humid chambers with a primary human anti-c-Kit antibody (1:150; rabbit polyclonal C-kit/CD117 antibody, DakoCytomation, Glostrup, Denmark). The sections were rinsed in PBS and incubated for $1 \mathrm{~h}$ at room temperature with a mixture of a horseradish peroxidase-conjugated goat anti-rabbit antibody (1:500; cat. 111-035-144, Jackson IR, West Grove, PA, USA). Histoenzymatic reaction was developed with 3,3'-diaminobenzidine (DAB-kit, cat. 34065, Pierce, Rockford, IL, USA) and slides were slightly counterstained with Mayer's haematoxylin. Finally, sections were dehydrated in alcohols, cleared with xylol and mounted in Entellan medium (Merck, Germany).

Double immunofluorescence staining. After antigen retrieval, sections were preincubated with normal serum as described above (hydrogen peroxide-blocking step was omitted). For the simultaneous visualisation of two antigens, an indirect double immunofluorescence procedure was used $[18,19]$. The sections were incubated overnight at room temperature in humid chambers with a mixture of primary antibodies: human anti-c-Kit (1:150; rabbit polyclonal C-kit) /CD117 antibody, DakoCytomation, Glostrup, Denmark) and human anti-mast cell tryptase (1:800, mouse monoclonal antibody, DakoCytomation, Glostrup, Denmark). The sections were rinsed in PBS and incubated for $1 \mathrm{~h}$ at room temperature with a mixture of a Cy3-conjugated goat anti-rabbit antibody (1:600; cat. 111-165-144, Jackson IR, West Grove, PA, USA) and a biotinylated goat anti-mouse antibody (1:600; cat. 115-065-146, Jackson IR, West Grove, PA, USA). The primary and secondary antibodies were diluted in the same solution as used in the pre-incubation step. After washing in PBS, the slides were incubated with DTAFconjugated streptavidin (1:500; cat. 016-010-084; Jackson IR, West Grove, PA, USA) for $1 \mathrm{~h}$. After a final rinse in PBS, the nuclei were counterstained with DAPI (1:30,000, D9542; SigmaAldrich, St. Louis, MO, USA) for 30 seconds. The sections were covered with a coverslip and Vectashield medium (H-1000; Vector Laboratories, Burlingame, CA, USA) to minimise photobleaching of fluorophores. Stained sections were examined with an Olympus BX50 brightfield/ 
/epifluorescence microscope (Olympus, Tokyo, Japan). For fluorescence observations, the microscope was equipped with mercuric burner and filter sets: U-MNG, U-MNIBA and U-MWA for the detection of Cy3 (red), DTAF (green) and DAPI (blue) fluorescence, respectively. Images were recorded using an Olympus DP71 digital CCD camera controlled by Olympus AnalySIS FIVE software, stored as TIFF files and analysed using Java ImageJ [20].

The use of mast cell tryptase staining allowed us to identify and distinguish c-Kit-positive mast cells from ICLCs and highlighted the morphological variability of mast cells. The potential misidentification of ICLCs and mast cells was prevented by performing only c-Kit immunohistochemical. For the assessment of immunohistochemical reaction (CD117 and mast cell tryptase) specificity, human ileum and GIST (gastrointestinal stromal tumour) archival paraffin blocks were used as control material (positive control).

\section{Results}

Histopathological examination exhibited traits of mild chronic cholecystitis in all patients enrolled in this study (Figure 1A). Immunoperoxidase staining allowed only the detection of numerous CD117-immunopositive cells in all layers of the gallbladder's wall (Figure 1B). The double immunofluorescence protocol was used to specifically identify ICCs/ICLCs. These cells were defined as c-Kit-immunopositive nucleated cells that lacked mast cell tryptase co-expression (tryptase-immunonegative). The presence of such cells was confirmed in reference material - ileum and GIST (Figure 1C and D, respectively). In gallbladders, ICLCs were predominantly fusiform in shape with branches that were visible in some sections. ICLCs were observed throughout the gallbladder, including the fundus, body (corpus) and neck, although they were mostly located in the body. The ICLCs were detected almost exclusively within the muscularis propria and were located primarily parallel to smooth muscle cells. However, in a few cases, the ICLCs were localised within the connective tissue separating the smooth muscle bundles. Thus, the following subpopulations of ICLCs were observed: ICLC-IM (intramuscular ICC) localised between smooth muscle fibres forming one muscle bundle; and ICLC-IB (interbundle ICLCs) localised within the connective tissue separating smooth muscle bundles (Fig. 1E and F). The ICLCs were generally sparsely distributed in other parts of the gallbladder's wall. They typically appeared individually or in small clusters of 2-3 cells, but they did not form a global network as was clearly seen in the small intestine (Figure 1C).

\section{Discussion}

The aim of this study was to determine whether interstitial Cajal-like cells exist in the human adult gallbladder and to identify their distinctive immunophenotypic characteristics. Generally, ICCs are difficult to identify. The classical identification of ICCs in the GI tract has been based on their ultrastructural characteristics [21]. Recently c-Kit receptor tyrosine kinase immunolocalisation has been used as a reliable marker of ICC presence in the GI tract and ICLCs in other organs $[8,11,21]$. In this study, immunohistochemistry was used to identify c-Kit immunopositive interstitial Cajal-like cells. However, mast cells also show c-Kit immunoreactivity and are abundant in the wall of the human gallbladder. Therefore, we used simultaneous anti-mast cell tryptase detection to better distinguish mast cells from ICLCs. Morphological characteristics of ICC have been shown to be species- and tissue-dependent, and have been divided into different classes according to their location in the GI tract [16].

In our specimens, the ICLCs were detected almost exclusively within the muscularis propria. They typically appeared individually or in small clusters of 2-3 cells, but they did not form a global network. However, the ICLCs/ICCs networks are best seen in whole mounts (2D-3D visualisation), therefore the failure to see networks is due to the nature of preparations used (thin paraffin sections), which did not allow for the determination of this architectural organisation.

The reports concerning ICLC distribution and number are conflicting for many reasons including: difficulties in visualising ICLCs in formalin-fixed, paraffin-embedded tissues despite HIER, proper human tissue handling (e.g. fixatives), the use of antibodies with varying sensitivities, and the lack of reliable quantification protocols. Establishing control values and standardised protocols for tissue collection, fixation, and ICLC visualisation is required [22].

Although we have documented the presence of interstitial Cajal-like cells in the human gallbladder, their function still remains unknown. ICLCs have been proposed to play a role as pacemakers in many organs. The existence of ICLCs in the gallbladder suggests that these cells may be involved in pacing electrical rhythmicity, imposing presumably the rhythm of bile release. We hypothesise that they could have a function similar to that of ICC in the gut. Indeed, there is growing evidence that the gallbladder's ICLCs are responsible for these processes and that their function may be influenced by numerous pathological factors (e.g. cholesterol in gallstone disease) 

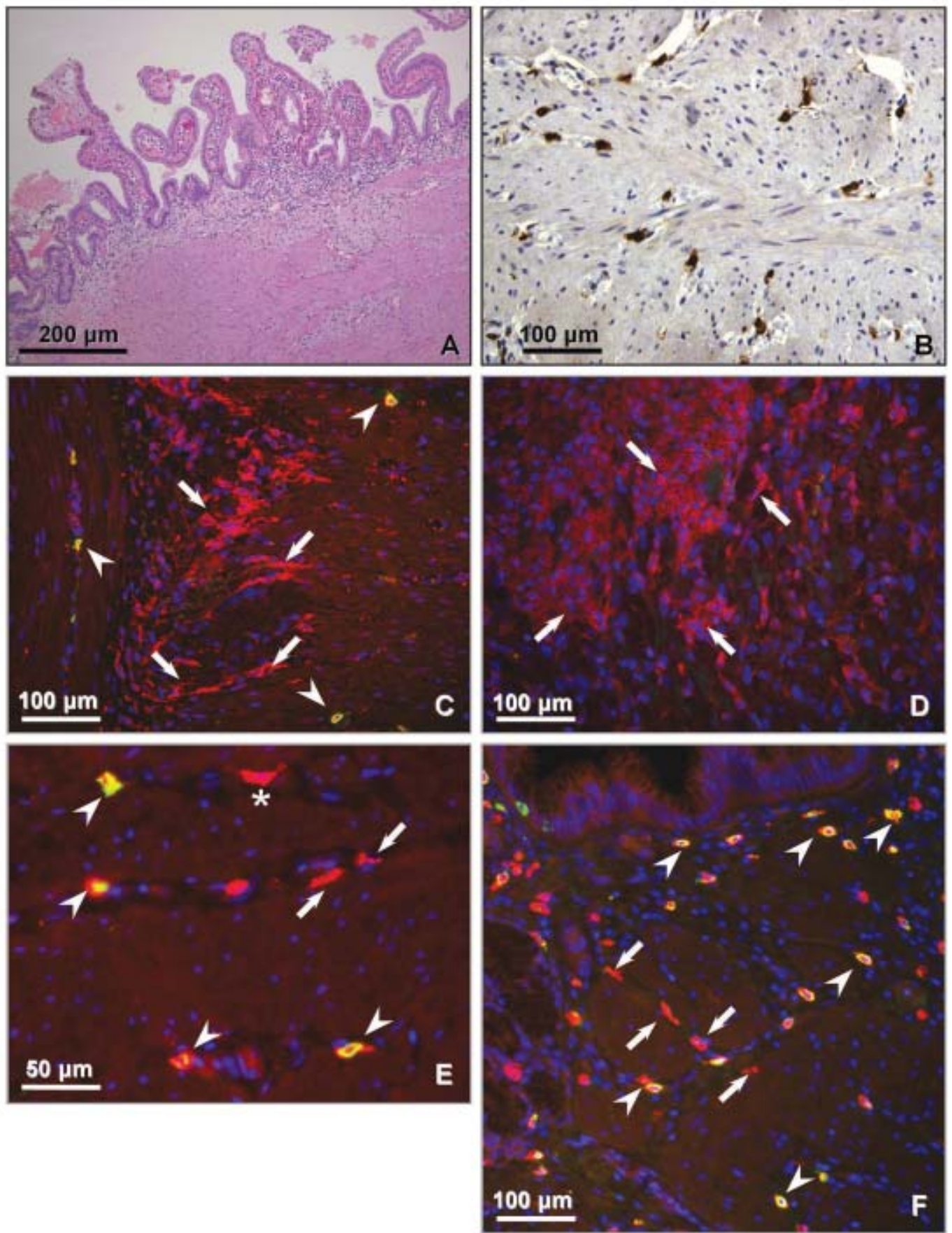

Figure 1. Sections of human ileum, gastrointestinal tumour and gallbladders stained by HE, immunoperoxidase and immunofluorescence methods to present ICCs/ICLCs and mast cells. In Figures C-F, CD117-immunopositive reactions were shown by red, tryptase-positive by green colour and cell nuclei were counterstained with DAPI (blue). A. Wall of the human gallbladder, mild chronic inflammation. H\&E staining, total magnification $100 \times$. B. Muscularis propria of human gallbladder stained for c-Kit (immunoperoxidase reaction). Note the numerous c-Kit-immunopositive cells (brown); $400 \times$. C. Muscularis propria from human ileum (positive control). Numerous c-Kit-positive and tryptase-negative interstitial Cajal cells (ICCs, red colour) forming a complex network are present (arrows). Few mast cells (c-Kit-positive and tryptase-positive) are indicated by arrowheads; $400 \times$. D. Section of gastrointestinal stromal tumour (GIST, positive control) shows numerous c-Kit-positive and tryptase-negative cells (arrows); $400 \times$. E. Muscularis propria of human gallbladder. Numerous c-Kit-positive and tryptase-negative ICLCs are located in the connective tissue separating smooth muscle bundles (ICLC-IB, arrows) and are seen between smooth muscle cells (asterisk). Mast cells are indicated by arrowheads; magnification $400 \times$. F. Cross-section of gallbladder wall presents inter-bundle ICLCs (ICLC-IB) marked with arrows. Mast cells are indicated by arrowheads; $400 \times$ 
[23]. Elucidation of the role of interstitial Cajal-like cells in the human gallbladder may contribute to new treatment options for gallbladder motility disorders, present in gallstone disease, for example.

In conclusion, interstitial Cajal-like cells were found throughout the wall of the human gallbladder and were present mainly within the muscularis propria. Double immunofluorescence using anti-CD117 and anti-mast cell tryptase antibodies represents a reliable method of identifying ICLCs and differentiating them from the numerous mast cells present in the gallbladder's wall.

\section{References}

1. Cajal SR. Nuevas aplicaciones del metodo de coloracion de Golgi. Gaceta Medica Catalana 1889;12:613-616.

2. Cajal SR. Sur le ganglions et plexus nerveux de l'intestin. $C R$ Soc Biol Paris. 1893;5:217-223.

3. Faussone-Pellegrini MS, Cortesini C, Romagnoli P. Ultrastructure of the tunica muscularis of the cardial portion of the human esophagus and stomach, with special reference to the so-called Cajal's interstitial cells. Arch Ital Anat Embriol. 1977;82:157-177.

4. Faussone-Pellegrini MS, Thuneberg L. Guide to the identification of interstitial cells of Cajal. Microsc Res Tech. 1999;47:248-266.

5. Thuneberg L. Interstitial cells of Cajal: intestinal pacemaker cells? Adv Anat Embryol Cell Biol. 1982;71:1-130.

6. Lammie A, Drobnjak M, Gerald W, Saad A, Cote R, Cordon-Cardo C. Expression of c-kit and kit ligand proteins in normal human tissues. J Histochem Cytochem. 1994;42: 1417-1425.

7. Iino S, Horiguchi K. Interstitial cells of cajal are involved in neurotransmission in the gastrointestinal tract. Acta Histochem Cytochem. 2006;39:145-153.

8. Popescu LM, Hinescu ME, Ionescu N, Ciontea SM, Cretoiu $\mathrm{D}$, Ardelean C. Interstitial cells of Cajal in pancreas. J Cell Mol Med. 2005;9:169-190.

9. Bassotti G, Villanacci V, Maurer CA et al. The role of glial cells and apoptosis of enteric neurones in the neuropathology of intractable slow transit constipation. Gut. 2006;55: 41-46.

10. Bassotti G, Battaglia E, Bellone G. Interstitial cells of Cajal, enteric nerves, and glial cells in colonic diverticular disease. J Clin Pathol. 2005;58:973-977.
11. Hinescu ME, Gherghiceanu M, Mandache E, Ciontea SM, Popescu LM. Interstitial Cajal-like cells (ICLC) in atrial myocardium: ultrastructural and immunohistochemical characterization. J Cell Mol Med. 2006;10:429-443.

12. Radu E, Regalia T, Ceafalan L, Andrei F, Cretoiu D, Popescu LM. Cajal-type cells from human mammary gland stroma: phenotype characteristics in cell culture. J Cell Mol Med. 2005;9:748-752.

13. Lang RJ, Klemm MF. Interstitial cell of Cajal-like cells in the upper urinary tract. J Cell Mol Med. 2005;9:543-556.

14. Ciontea SM, Radu E, Regalia T, et al. C-kit immunopositive interstitial cells (Cajal type) in human myometrium. J Cell Mol Med. 2005;9:407-420.

15. Parr EJ, Kennedy AL, Mawe GM. Lack of evidence for the existence of interstitial cells of Cajal in the gallbladder. Gastroenterology 2003;124:347.

16. Lavoie B, Balemba OB, Nelson MT, Ward SM, Mawe GM. Morphological and physiological evidence for interstitial cell of Cajal-like cells in the guinea pig gallbladder. J Physiol. 2007;579:487-501.

17. Sun X, Yu B, Xu L, Dong W, Luo H. Interstitial cells of Cajal in the murine gallbladder. Scand J Gastroenterol. 2006;41: $1218-1226$.

18. Kasacka I, Łebkowski W, Janiuk I, Łapińska J, Lewandowska A. Immunohistochemical identification and localisation of gastrin and somatostatin in endocrine cells of human pyloric gastric mucosa. Folia Morphol (Warsz). 2012;71: $39-44$.

19. Gajda M, Jawień J, Mateuszuk $\succeq$ et al. Triple immunofluorescence labeling of atherosclerotic plaque components in apoE/LDLR -/- mice. Folia Histochem Cytobiol. 2008;46: 143-146.

20. Mizia E, Tomaszewski KA, Lis GJ, Goncerz G, Kurzydło W. The use of computer-assisted image analysis in measuring the histological structure of the human median nerve. Folia Morphol (Warsz). 2012;71:82-85.

21. Popescu LM, Faussone Pellegrini MS. TELOCYTES a case of serendipity: the winding way from Interstitial Cells of Cajal (ICC), via Interstitial Cajal-Like Cells (ICLC) to TELOCYTES. J Cell Mol Med. 2010;14:729-740.

22. Huizinga JD, Zarate N, Farrugia G. Physiology, injury, and recovery of interstitial cells of Cajal: basic and clinical science. Gastroenterology. 2009;137:1548-1556.

23. Lavoie B, Nausch B, Zane EA et al. Disruption of gallbladder smooth muscle function is an early feature in the development of cholesterol gallstone disease. Neurogastroenterol Motil. 2012;24:e313-324.

Submitted: 19 September, 2012 Accepted after reviews: 7 November, 2012 